\author{
D.J. Edell, V.M. McNeil, M.G. Curley, J.H. Wolfe * \\ Massachusetts Institute of Technology \\ Harvard-MIT Division of HST \\ Cambridge, Massachusetts 02139
}

Electrical and chemical micro-sensors for biological cell research allow for the continuous study of biological systems under "normal" physiological conditions. Two sensor technologies which take particular advantage of microfabrication technology will be discussed in this paper. One technology is being developed for monitoring the environment of cancer cells during radiotherapy, chemotherapy, and hyperthermia treatments. Of current interest is the measurement of temperature and interstitial free oxygen concentration distributions in cancer tissues prior to and during various treatments. The ability to fabricate small microstructures with well controlled geometries which allow repeatable, accurate measurements in a wide variety of tissues is being exploited in this research. The second technology to be discussed is being developed for monitoring the extracellular ionic currents from electrogenic cells in culture. The ability to build integrated circuits over large areas of a silicon wafer which can impedance transform the signals and multiplex a large array of contacts is being used in this research.

Many parameters can affect the response of cancer cells to radiotherapy, chemotherapy and hyperthermia treatments [1]. The dissolved oxygen concentration in tumor tissues is one important parameter which seems to be strongly related to the destruction of cancer cells during radiotherapy, and may also affect the response of cells to hyperthermia and chemotherapy. oxygen concentration and temperature in normal tissue and cancer tumors varies widely over small distances. Accurate, multipoint measurements within the tumor of interest are essential for research and clinical therapies. Since the local environment of the sensor will be highly variable due to tissue property variations, a measurement technology which is independent of the environment is essential. Also, since the probes are to be inserted into living tissue, the size of the probe must be minimized.

The prototype probe being developed for cancer research is $1 \mathrm{~mm}$ wide, $0.25 \mathrm{~mm}$ thick, and $4 \mathrm{~cm}$ long. Oxygen sensors on the probe use the multicathode amperometric approach $[2,3]$ implemented on a micron scale. The oxygen electrode design takes advantage of the hemispherical steady state oxygen diffusion field which forms about a very small, properly polarized hemispherical cathode on an insulating substrate. The diffusion field limits the transport of oxygen to the cathode surface and hence limits the current flow through the cathode. The steady state oxygen concentration about the cathode is given by:

where:

$$
c_{x}=c_{0}(1-r / x)
$$

$c x=$ oxygen concentration at $x$

$\mathrm{C}_{\mathrm{o}}^{\mathrm{x}}=$ oxygen concentration at infinity

$r^{\circ}=$ cathode radius

$x=$ distance from cathode center

The steady state oxygen current about the hemispherical cathode is given by:

where:

$$
I=-8 q D r C_{0}
$$

$I=$ oxygen current

$\mathrm{C}_{\mathrm{o}}=$ oxygen concentration at infinity

$\mathbf{r}=$ cathode radius

$D=$ oxygen diffusivity

$\mathrm{q}=$ electron charge
By making the cathode radius very small, the oxygen concentration a small distance from the cathode is minimally disturbed which implies that the diffusion field surrounding the cathode is limited in extent. By coating the surface of the cathode with a layer of a material of known and stable characteristics, diffusion of oxygen to the cathode will be independent of the environment with the exception of variations in temperature. Temperature can be compensated for by incorporation of temperature sensors under the diffusion film on the substrate surface.

Since the magnitude of the oxygen current is proportional to the radius of the electrode, microcathodes which have very limited diffusion fields produce very low, difficult to resolve currents. Numerous microcathodes, operating in parallel and appropriately spaced to avoid significant interaction, will produce a steady state oxygen current of reasonable amplitude.

Detailed analysis of the diffusion fields of the multi-microcathode sensor is difficult and probably not necessary. However, considerable design insight can be derived from a simple, approximate model of the structure. The microcathodes actually fabricated lie at the base of a hole in the dielectric coating. $0 x y-$ gen concentration gradients in these holes are assumed to be linear. At the surface of the dielectric, the oxygen concentration gradients are assumed to be hemispherical. Even though the microcathodes are spaced 20 radil apart, together they form a large area sink for oxygen current. Oxygen concentration gradients about the ensemble of microcathodes again are treated as hemispherical.

Results of the analysis show that a hemispherical microcathode would cause only a $5 \%$ depletion of the oxygen concentration 20 radii from the center of the microcathode in the absence of a well. The presence of a dielectric with a thickness equal to the diameter of the microcathode creates a microwell which reduces the oxygen depletion at 20 radii to about 1\%. Approximate analysis of the ensemble of microcathodes was done assuming a 20 radii spacing of microcathode and a fixed area on which to place the microcathode. Shrinking the radius of each microcathode reduces the oxygen current per microcathode as $\mathrm{r}^{-1}$, but at the same time, more microcafhodes per unit area increases the oxygen current as $\mathrm{r}^{2}$. Thus, the optimal microcathode size is as small as possible until narrow channel transport effects dominate.

The performance of the oxygen sensors is in general agreement with the predictions. Results using uncoated sensors show typical polarographic responses and rapid electrical transient response times. Prototype sensors which have 506 two micron diameter micro-cathodes exhibit responses which are nearly flow independent which indicates a very limited diffusion field. Results of similar measurements using a single large cathode geometry on the same substrate shows a substantial flow dependence. Work on coating the sensors with thin films to stabilize the response to variations in local environment is under way. 


\section{Cell Culture Arrays}

The study of the electrical activity of brain and heart cells can sometimes be simplified by culturing cells from these organs onto planar recording electrode arrays. This technique is ideally suited for studying the electrical interactions between neural [4] and cardiac [5] cells in a relatively controlled, two dimensional environment. The standard technique for producing arrays of electrodes for recording from cell culture preparations is to define the electrode and lead pattern on a glass substrate and use a polymer coating for a dielectric [6]. Typically ten to forty contacts are defined this way. Each contact must then be wired to the preamplifiers and data acquisition system. The most typical technique for accomplishing this is ultrasonic bonding of small gold wires.

Since electrode contact areas are typically small, the source impedance for the acquired signals is usually 0.1-10 megohms. While careful instrumentation design can minimize noise pickup in the high impedance leads, capacitive shunting of the signal and the number of electrode connections which have to be individually connected to external electronics limits the utility of the technology.

We are developing a monolithic array of independently addressable MOSFET sensors for use in monitoring the electrical activity of cells in culture. The gate of each MOSFET is connected to gold contacts on the surface of the silicon wafer for recording from nearby cells. Devices are selected by applying power to the drain bus of a particular row and recording from the appropriate source bus of a particular column. Electrical potentials created by ionic current flow in the vicinity of the gold electrode contacts create signals on the gates of the MoSFETs. The low impedance output of each device reduces signal attenuation due to shunt capacitances, and minimizes cross talk and noise pickup.

The cell culture recording matrix under development provides access to 256 recording sites and requires only 32 connections to the source and drain buses plus two connections for substrate bias and a solution reference. Using 16 channel parallel recording from the 16 source buses, it is possible to rapidly scan the array by stepping power through the 16 drain buses.

\section{* Acknowledgements}

This paper discusses results from two research efforts which each have a large number of participants. The hyperthermia project is sponsored by NIHNCI Grant \#CA37235, and involves H.F. Bowman, S.K. Burns, D.J. Edell, J.C. Weaver, M.G. Curley, L.M. Peznola, and J.H. Wolfe. The cell culture array is unsponsored research which at various times has involved D.J. Edell, R.G. Mark, M.S. Cafferty, L.D. Clark, D.A. Israel, V.M. McNeil, and P.M. Wheaton.

\section{References}

1. Oleson, J.R. and M.W. Dewhirst, Hyperthermia: An overview of current progress and problems. In: Hickey, R.C. (ed) Current Problems in Cancer, 8: 6. Chicago:Year Book Medical Publishers, 1983.

2. Fatt, I. and R. St. Helen, A multicathode polarographic oxygen sensor and its performance, J. Appl. Biol. 27, 435-437, 1969.

3. Siu, W., and R.S.C. Cobbold, Characteristics of a multicathode polarographic oxygen electrode, Med. Bio. Eng. 14, 109-120, 1976.
4. Thomas, CA, Springer, PA, Loeb, GE, Berwald-

Netter,Y, Okun, LM. A miniature microelectrode array to monitor the bioelectric activity of cultured cells. Exptl. Cell. Res. Vol. 74: 61-66, 1972.

5. Shtark, MB, AS Ratushnyak, LV Voskresenskaya, and SN Olenev. A multi-electrode perfusion chamber for tissue culture research. Bull. Exp. Biol. Med. USSR 78: 1090-1092, 1974.

6. Gross, GW. Simultaneous single unit recording IN VITRO with a photoetched laser deinsulated gold multimicroelectrode surface. IEEE Trans. Biomed. Eng. BME-26: 273-279, 1979.

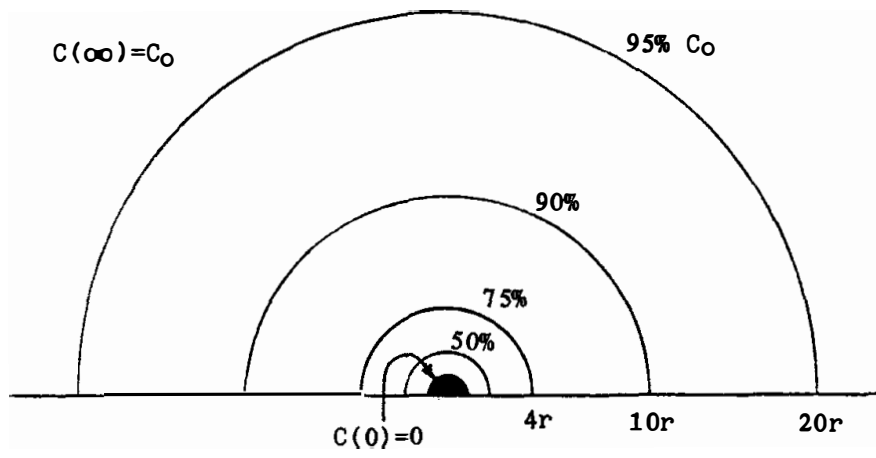

Oxygen Concentration Field for a Cathode of Radius $r$

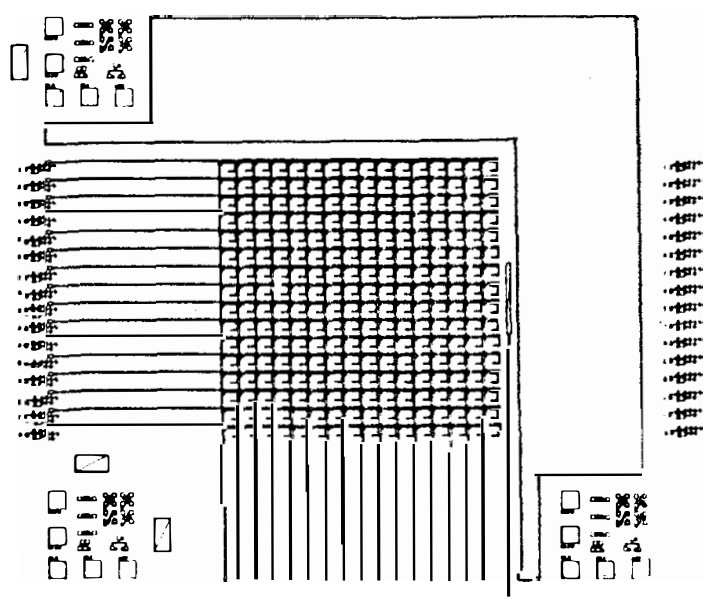

Cardiac Cell Culture Array

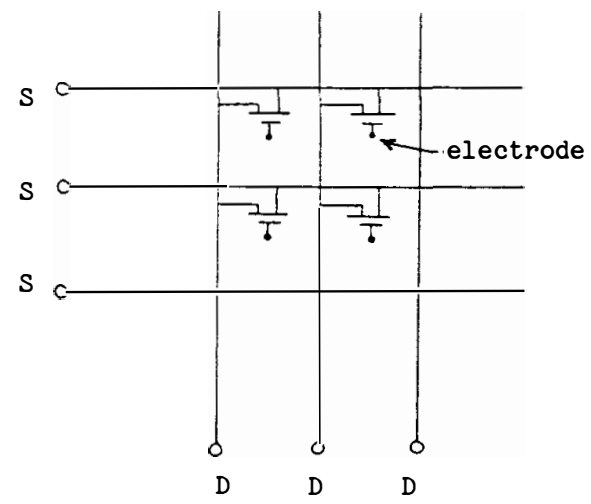

Schematic Closeup of Cell Culture Array 